\title{
Risco de fragilização e uso de medicamentos em idosos residentes em uma localidade do sul de Santa Catarina
}

Frailty risk and drug use among elderly in southern Santa Catarina state, Brazil

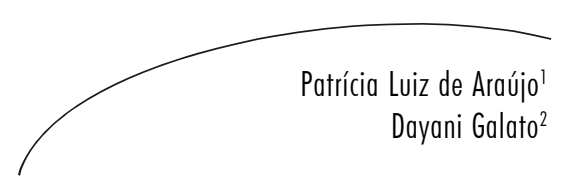

Resumo

Objetivo: Conhecer o risco de fragilização (repetidas internações hospitalares) e o perfil de utilização de medicamentos em idosos vinculados ao Serviço de Assistência Integral à Saúde da Universidade do Sul de Santa Catarina, localizado na cidade de Tubarão. Métodos: Em 2009, quando foram coletadas as informações, 772 idosos estavam cadastrados pelos agentes comunitários vinculados ao serviço, e 135 fizeram parte da amostra desta pesquisa. Para a seleção dos participantes, as residências dos idosos foram distribuídas geograficamente. A coleta de dados ocorreu através de entrevistas realizadas no ambiente domiciliar. Os resultados foram apresentados por estatística descritiva, sendo posteriormente adotado o teste do qui-quadrado $(\mathrm{p}<0,05)$ para determinar os fatores associados. Resultados: Dos entrevistados, $65,9 \%$ possuíam até três problemas de saúde e $21,5 \%$ eram polimedicados, sendo os medicamentos mais comuns aqueles pertencentes ao sistema cardiovascular e ao sistema nervoso. Utilizaram antibióticos no último mês $14,3 \%$ dos entrevistados e $17,0 \%$ dos idosos estavam utilizando medicamentos considerados potencialmente impróprios. Dos idosos, 29,2\% afirmaram adotar a prática da automedicação e 64,4\% referiram utilizar plantas medicinais. Quando avaliado o risco de fragilização desta população, 88,1\% foram classificados como baixo risco. Associaram-se ao maior risco de fragilização a polimedicação $(\mathrm{p}<0,001)$, possuir mais que três problemas de saúde $(\mathrm{p}<0,001)$ e o fato de ter utilizado antibiótico no último mês $(\mathrm{p}=0,015)$. Conclusões: Os idosos em ambiente domiciliar apresentam baixo risco de fragilização e o maior risco está associado a presença de múltiplos problemas de saúde, polimedicação e uso de antibiótico no último mês.

\footnotetext{
Curso de Farmácia. Núcleo de Pesquisa em Atenção Farmacêutica e Estudos de Utilização de Medicamentos. Universidade do Sul de Santa Catarina - Unisul. Tubarão, SC, Brasil.

2 Programa de Pós-Graduação em Ciências da Saúde e Curso de Farmácia. Núcleo de Pesquisa em Atenção Farmacêutica e Estudos de Utilização de Medicamentos. Universidade do Sul de Santa
} Catarina - Unisul. Tubarão, SC, Brasil.

Correspondência / Correspondence

Dayani Galato

Programa de Pós-Graduação em Ciências da Saúde - Unisul

Av. José Acácio Moreira, 787 - Bairro Dehon

88704-900 Tubarão, SC, Brasil

E-mail: dayani.galato@unisul.br
Palavras-chave: Idoso. Saúde do idoso. Uso de medicamentos. Medicamentos. 


\section{Abstract}

Objective: To examine frail risk (repeated hospital admissions) and the profile of drug use among elderly attending the Integrated Health Service of the University of Southern Santa Catarina State, in the city of Tubarão. Methods: When the information was collected by the community service agents in 2009 , there were 772 elderly registered, and 135 were included in the research sample. The geographical distribution of residences for the elderly was taken into account for the selection of the participants in the survey. Data was collected in household interviews. Descriptive statistics was used to present the results. The Pearson chi-square test $(\mathrm{p}<0.05)$ was used to test associated factors. Results: In total, 135 elderly were interviewed, $65.9 \%$ of whom had up to three concomitant health problems and $21.5 \%$ received multiple medications. Cardiovascular system and nervous system medications were the most commonly used. Of the respondents, $14.3 \%$ had used antibiotics in the last month and $17.0 \%$ were using medications considered potentially inappropriate. Self-medication was reported by $29.2 \%$ of the elderly and $64.4 \%$ reported use of medicinal plants. With regard to frailty risk, $88.1 \%$ were classified as low. The following were risk factors associated with frailty: polymedication $(\mathrm{p}<0.001)$, having more than three concomitant health problems $(\mathrm{p}<0.001)$, and having used antibiotics in the last month $(\mathrm{p}=$ 0.015). Conclusions: The elderly who live at their own home are at low risk for frailty. Frailty risk is associated with the presence of multiple health problems, polymedication and recent use of antibiotics.
Key words: Elderly. Elderly health care. Drug use. Medication.

\section{INTRODUÇÃO}

O maior número de idosos na população resulta em um acréscimo na utilização de serviços de saúde devido a problemas crônicos, tendo como consequência intervenções de alto custo e de tecnologia específica para o cuidado necessário ao idoso, como o aumento de internações hospitalares (risco de fragilização) e do consumo de medicamentos, que leva à polimedicação. ${ }^{1}$ Segundo Lojoduci e colaboradores, ${ }^{2}$ o uso de cinco ou mais medicamentos simultaneamente caracteriza a polimedicação.

A polimedicação pode levar ao aparecimento de problemas relacionados com os medicamentos ${ }^{3} \mathrm{e}$ uma solução viável é aumentar a colaboração entre os profissionais da saúde, contribuindo para aumentar a adesão ao tratamento e as prescrições adequadas, diminuir os efeitos nocivos dos medicamentos e promover o uso e o armazenamento de medicamentos de forma correta. ${ }^{4}$

Outro ponto importante que merece atenção é o fato de que, na terceira idade, ocorrem alterações fisiológicas e surgem morbidades que tornam alguns medicamentos impróprios ao paciente..$^{5}$ Estas alterações fisiológicas também propiciam o aparecimento de infecções e, consequentemente, o uso de antibióticos, o que merece também uma cautela especial. ${ }^{6}$ No entanto, mesmo com estas alterações fisiológicas e o uso combinado de medicamentos de uso contínuo, os idosos ainda praticam a automedicação, geralmente para problemas de saúde considerados simples e estimulados por amigos e familiares. A praticidade é um dos motivos mais citados para a automedicação, visto que muitas vezes limitações impedem que os idosos se desloquem até os serviços de saúde.?

Esses são alguns dos problemas que podem levar os idosos a uma fragilização e probabilidade de agravos maiores. Contudo, se esses fatores fossem identificados precocemente e de forma preventiva em serviços de menor complexidade, haveria a possibilidade de se gastar menos em hospitalizações. ${ }^{1,8}$ Nesse contexto, o risco de fragilização determina a chance de hospitalização do paciente, cujo cálculo foi desenvolvido por Pacala et. al. ${ }^{9}$ e adotado no Brasil por Veras. ${ }^{1}$ 
Desta forma, este trabalho teve por objetivo identificar o risco de fragilização e o perfil de utilização de medicamentos de idosos residentes na área de cobertura do Serviço de Assistência Integral à Saúde (SAIS) sediado na Universidade do Sul de Santa Catarina.

\section{METODOLOGIA}

Trata-se de estudo de caráter exploratório, transversal baseado na técnica de entrevista. A população do estudo foi representada por indivíduos residentes na área de cobertura do SAIS, sediado na Universidade do Sul de Santa Catarina e vinculado à Secretaria Municipal de Saúde de Tubarão.

O SAIS é formado por duas Equipes da Estratégia de Saúde da Família, onde estão inscritos 15 agentes comunitários de saúde. Em 2009, quando os dados foram coletados, havia 772 idosos cadastrados por esses agentes. Para o cálculo da amostra, adotaram-se um erro estimado de 5,0\%, um nível de confiança de $95 \%$ e uma prevalência de risco de fragilização de $11,0 \%$, segundo dados apresentados por Veras. ${ }^{1}$

Para a seleção dos idosos participantes na pesquisa, realizou-se a distribuição geográfica das residências, feita proporcionalmente em cada uma das 15 áreas de cobertura dos agentes de saúde.

A coleta de dados foi realizada nos domicílios dos idosos previamente selecionados. Inicialmente, os agentes de saúde informaram os idosos selecionados sobre a pesquisa. No dia da entrevista, o idoso ou seu cuidador (quando esse estava impossibilitado de se comunicar) foi abordado na residência para a apresentação formal da pesquisa. Aceitando participar, o idoso ou cuidador assinou o termo de consentimento livre e esclarecido e participou da entrevista.

Foi adotado um roteiro estruturado que permitiu a coleta de dados a respeito das características sócio-demográficas (sexo e idade), nosológicas (problemas de saúde, quedas no último ano, fraturas em função de quedas), de utilização de medicamentos (tipos de medicamentos, número de medicamentos, adoção da automedicação, de plantas medicinais e uso de antibiótico no último mês) e dados relacionados a fragilização, segundo descrito por Veras, ${ }^{1}$ tais como autopercepção de saúde, número de hospitalizações e consultas médicas no último ano, diagnóstico de diabetes e problema cardiovascular no último ano e presença de apoio familiar. Destaca-se que, no ano de 2005, Dutra e colaboradores validaram este instrumento de determinação do risco de fragilização, aplicandoo a idosos da cidade de Progresso, no estado do Rio Grande do Sul, Brasil. ${ }^{10}$

Os medicamentos em uso foram classificados segundo a Anatomical Therapeutic Chemical Classification (ATC). ${ }^{11}$ Para a avaliação dos medicamentos considerados impróprios, independentemente de diagnóstico ou condições do idoso, adotou-se a lista de medicamentos elaborada por Fick e colaboradores. ${ }^{5}$

Para o cálculo do fator de risco de fragilização, foram adotados os parâmetros definidos por Pacala e colaboradores ${ }^{9}$ e a escala descrita por Veras ${ }^{1}$. Esta determina a Probabilidade de Admissão Repetida em um período de quatro anos ( $P A R)$ : quando $P A R$ for menor que 0,300 o risco é baixo; de 0,300 a 0,399 é médio; de 0,400 a 0,499 é médio alto; e maior que 0,500 , muito alto.

Para análise dos dados, foi desenvolvido um banco de dados no programa EpiData e posteriormente realizadas as análises estatísticas no programa SPSS 19.0. As variáveis nominais foram apresentadas em valores absolutos e relativos; e as variáveis numéricas, em medida de tendência central e de dispersão. Para avaliar os fatores associados ao risco de fragilização e a polimedicação, foi realizado o teste do quiquadrado, adotando-se $\mathrm{p}<0,05$ como significante. No caso de variáveis numéricas, as mesmas foram categorizadas para a análise bivariada adotandose a mediana.

O projeto foi aprovado pelo Comitê de Ética e Pesquisa da Universidade do Sul de Santa Catarina sob o código 06.458.4.03.III. 


\section{RESULTADOS}

Foram entrevistados 135 idosos com idade entre 60 e 93 anos $(72,4 \pm 7,29)$, sendo que $62,2 \%$ foram representados por mulheres.

Foi referida a presença de problemas de saúde por $128(94,8 \%)$ idosos, com uma média de 3,0 $( \pm 1,87)$ problemas por idoso, variando de zero a nove problemas. Os problemas de saúde referidos mais prevalentes foram hipertensão arterial sistêmica $(51,8 \%)$, labirintite $(25,2 \%)$, diabetes $(22,9 \%)$, depressão $(21,5 \%)$ e ansiedade $(21,5 \%)$.
Quando indagados a respeito de quedas no último ano, 42 (31,3\%) referiram ter sofrido; destes, dez $(20,8 \%)$ apresentaram fraturas em função das referidas quedas.

Aplicando-se a questões sugeridas por Veras ${ }^{1}$ sobre os fatores de risco de admissão hospitalar, foram obtidas as respostas apresentadas na tabela 1. Quanto ao grau de risco calculado segundo Pacala et. al., observou-se que $88,1 \%$ dos idosos estão classificados no baixo risco.

Tabela 1 - Distribuição dos fatores de risco para internação hospitalar. Tubarão, SC, 2009.

\begin{tabular}{|c|c|c|}
\hline Fator de Risco & $\mathrm{n}$ & $\%$ \\
\hline \multicolumn{3}{|l|}{ Autopercepção de saúde $(\mathrm{N}=134)$} \\
\hline Excelente & 15 & 11,2 \\
\hline Muito boa & 51 & 38,1 \\
\hline Boa & 49 & 36,6 \\
\hline Média & 16 & 11,9 \\
\hline Ruim & 3 & 2,2 \\
\hline \multicolumn{3}{|l|}{ Hospitalizações nos últimos 12 meses $(\mathrm{N}=105)$} \\
\hline Nenhuma vez & 85 & 81,0 \\
\hline $1 \mathrm{vez}$ & 14 & 13,3 \\
\hline 2 ou mais & 6 & 5,7 \\
\hline \multicolumn{3}{|c|}{ Consultas médicas nos últimos 12 meses $(\mathrm{N}=122)$} \\
\hline Nenhuma vez & 14 & 11,5 \\
\hline $1 \mathrm{vez}$ & 21 & 17,2 \\
\hline 2 ou 3 & 46 & 37,7 \\
\hline 4 a 6 & 30 & 24,6 \\
\hline Mais de 6 & 11 & 9,0 \\
\hline \multicolumn{3}{|c|}{ Diagnóstico de diabetes nos últimos 12 meses $(\mathrm{N}=127)$} \\
\hline Sim & 12 & 9,4 \\
\hline \multicolumn{3}{|c|}{ Diagnóstico de doença cardiovascular nos últimos 12 meses $(\mathrm{N}=128)$} \\
\hline Sim & 21 & 16,4 \\
\hline \multicolumn{3}{|l|}{ Sexo $(N=135)$} \\
\hline Masculino & 51 & 37,8 \\
\hline \multicolumn{3}{|l|}{ Apoio Social ${ }^{a}(\mathrm{~N}=135)$} \\
\hline $\operatorname{Sim}$ & 127 & 94,1 \\
\hline \multicolumn{3}{|l|}{ Distribuição por faixa etária $(\mathrm{N}=134)$} \\
\hline $60-64$ & 20 & 14,9 \\
\hline 65- 74 & 64 & 47,8 \\
\hline $75-79$ & 25 & 18,6 \\
\hline $80-84$ & 19 & 14,2 \\
\hline$\geq 85$ & 6 & 4,5 \\
\hline \multicolumn{3}{|l|}{ Grau de $\operatorname{risco}^{\mathrm{b}}(\mathrm{N}=135)$} \\
\hline Baixo & 119 & 88,1 \\
\hline Médio & 12 & 8,9 \\
\hline Médio/alto & 4 & 3,0 \\
\hline Muito alto & 0 & 0,0 \\
\hline
\end{tabular}

${ }^{2}$ Apoio Social: disponibilidade de cuidados por familiares, amigos e vizinhos.

'Segundo os parâmetros de Pacala et al. ${ }^{9}$ 
Quanto à utilização de medicamentos de uso contínuo, $117(86,7 \%)$ responderam afirmativamente, referindo utilizar entre um e 13 medicamentos, conferindo uma média de 3,5 $( \pm 2,25)$ medicamentos por idoso. Destes, 29 $(21,5 \%)$ foram classificados como polimedicados, ou seja, utilizavam cinco ou mais medicamentos simultaneamente.

Entre as classes terapêuticas mais frequentes estão: cardiovascular (42,3\%), onde se destacam a hidroclorotiazida, usada por $17,8 \%$ dos idosos, o captopril (11,8\%), o enalapril (10,4\%), o atenolol $(9,6 \%)$ e o losartan $(8,1 \%)$; sistema nervoso $(21,2 \%)$, onde se destacam o clonazepam $(7,4 \%)$ e o Ginkgo biloba (6,7\%); trato alimentar e metabolismo $(18,0 \%)$, tendo como principais representantes o carbonato de cálcio $(7,4 \%)$, o omeprazol (8,9\%), a metformina $(16,3 \%)$ e a glibenclamida (7,4\%); e sangue e órgão formadores de sangue, representando $6,9 \%$ dos medicamentos e cujo principal representante foi o ácido acetilsalicílico, utilizado por $15,6 \%$ dos idosos.

Quanto ao uso de antibiótico no último mês, 19 (14,3\%) idosos responderam afirmativamente, sendo que os mesmos foram indicados por um profissional prescritor em $89,5 \%$ das situações.

Segundo o critério de medicamentos potencialmente impróprios para uso em idosos, ${ }^{5}$ pode-se verificar que 13 medicamentos foram classificados desta forma (tabela 2), sendo utilizados por $23(17,0 \%)$ idosos. Dos idosos que sofreram quedas no último ano, três utilizavam benzodiazepínicos de longa duração.

Tabela 2 - Medicamentos potencialmente inapropriados utilizados pelos idosos segundo Fick et al. ${ }^{5}$ Tubarão, SC, 2009. ( $\mathrm{N}=27)$

\begin{tabular}{lcccc}
\hline $\begin{array}{l}\text { Medicamentos } \\
\text { inapropriados }\end{array}$ & Código ATC $^{\mathrm{a}}$ & $\mathrm{n}$ & $\%$ & Severidade $^{\mathrm{b}}$ \\
\hline Alprazolam & N05BA12 & 2 & 7,4 & Alta \\
Amiodarona & C01BD01 & 1 & 3,7 & Alta \\
Amitriptilina & N06AA09 & 3 & 11,2 & Alta \\
Clonidina & C02AC01 & 2 & 7,4 & Baixa \\
Diazepam & N05BA01 & 2 & 7,4 & Alta \\
Digoxina & C01AA05 & 1 & 3,7 & Baixa \\
Doxazosina & C02CA04 & 2 & 7,4 & Baixa \\
Ergotamina & N02CA02 & 1 & 3,7 & Baixa \\
Fluoxetina & N06AB03 & 4 & 14,8 & Alta \\
Lorazepam & N05BA06 & 2 & 7,4 & Alta \\
Metildopa & C02AB01 & 2 & 7,4 & Alta \\
Nifedipina & C08CA05 & 4 & 14,8 & Alta \\
Ticlopidina & B01AC05 & 1 & 3,7 & Alta \\
Total & & 27 & 100,0 & \\
\hline
\end{tabular}

a. Medicamentos classificados segundo a Anatomical Therapentic Chemical Classification - ATC. ${ }^{11}$

b. Severidade segundo Fick et al. ${ }^{5}$ 
Avaliando-se o uso de medicamentos por automedicação, $70,8 \%$ dos idosos afirmaram adotar esta prática e $64,4 \%$ referiram utilizar plantas medicinais. Quanto ao uso de plantas, 65 idosos citaram 44 diferentes plantas medicinais, sendo que as mais comuns foram a hortelã (24,6\%), o boldo (20,0\%), a camomila (20,0\%) e a erva cidreira (16,9\%). É importante salientar que não foi realizada avaliação botânica das plantas citadas pelos idosos.

Analisando-se os fatores relacionados ao risco de fragilização, identificou-se que o sexo $(p=0,981)$, a idade $(p=0,233)$, o uso de medicamentos inapropriados $(\mathrm{p}=0,367)$, a prática da automedicação $(p=0,275)$, a ocorrência de quedas $(0,486)$ e o uso de plantas medicinais $(\mathrm{p}=0,199)$ não se mostraram associados. Por outro lado, polimedicação $(\mathrm{p}<0,001)$, possuir mais que três problemas de saúde $(p<0,001)$ e ter usado antibiótico no último mês $(\mathrm{p}=0,015)$ associaramse de forma significativa ao risco de fragilização proposto por Pacala et. al. ${ }^{9}$

A polimedicação, por sua vez, não esteve associada ao sexo $(\mathrm{p}=0,201)$ e à idade dos entrevistados $(p=0,695)$.

\section{DISCUSSÃO}

O perfil dos idosos demonstra que esta população apresenta vários problemas de saúde, em especial problemas crônicos que necessitam de medicamentos de uso contínuo, com prevalência dos problemas cardiovasculares, como demonstrado em outros trabalhos. ${ }^{3,6,12,13}$ Este resultado destaca a importância de desenvolver ações educativas para a promoção de estratégias que visem à adesão terapêutica nestes problemas de saúde. ${ }^{14}$

Ao se verificar quais os medicamentos utilizados pelos idosos, 13 foram classificados como impróprios, totalizando $6,5 \%$, sendo que os mais frequentes foram o nifedipino, a fluoxetina e a amitriptilina. Em trabalho desenvolvido por Rozenfeld e colaboradores, ${ }^{15}$ identificou-se percentual um pouco maior de consumo destes medicamentos $(10 \%)$, sendo os mais comuns os relaxantes músculo-esqueléticos, anti-histamínicos e benzodiazepinicos. Esses medicamentos devem ser evitados pelos idosos, por apresentarem risco para essa população, uma vez que podem predispor a fragilização, o que não foi identificado nesta pesquisa.

Identificou-se que três dos idosos que utilizavam benzodiazepínicos de longa duração sofreram quedas no último ano. Apesar de não ter sido verificada relação direta entre o uso da medicação e a queda, sabe-se que estes medicamentos são potencialmente impróprios para uso em idosos por este motivo. Neste caso, o risco de quedas entre idosos que utilizam benzodiazepínicos tem sido atribuído a certas propriedades desses medicamentos, como a atividade sedativa, que pode causar alterações psicomotoras, e o bloqueio á-adrenérgico, que aumentaria a probabilidade de hipotensão postural. ${ }^{16}$

A porcentagem de utilização de antibióticos pela população do estudo $(14,3 \%)$ é muito semelhante a do estudo de Tibincoski e colaboradores $^{6}(12,9 \%)$. A utilização desses medicamentos por idosos acaba se tornando comum, pois em função da fragilidade clínica, há uma susceptibilidade maior a infecções, dado confirmado neste trabalho pela associação deste fator com o risco de fragilização $(p=0,015)$.

Neste estudo, observou-se a adoção da automedicação e de plantas medicinais. Essa prática deve ser modificada, pois assim como os medicamentos usados no autocuidado, as plantas também podem interagir com os medicamentos de uso contínuo, predispondo os pacientes a reações adversas e toxicidade, devendo ser utilizadas de forma racional para evitar prejuízos à saúde e possíveis interações. ${ }^{7}$

Em comparação ao estudo de Veras, ${ }^{1}$ no qual $75,8 \%$ dos idosos pesquisados apresentam baixo risco de adoecer, o presente estudo teve resultado ainda melhor, pois mostrou que quase $90 \%$ da população em questão foram classificados no baixo risco de fragilização - portanto, têm menor 
chance de sofrer admissão hospitalar. Esta diferença talvez esteja relacionada às diferenças metodológicas na seleção da amostra das duas pesquisas, ou seja, naquela de $\operatorname{Veras}^{1}$ a pesquisa foi realizada com idosos que procuraram o serviço ambulatorial (o que geralmente ocorre por problemas de saúde), enquanto no presente trabalho, os idosos foram entrevistados no ambiente domiciliar. Neste caso, ambiente domiciliar, é maior a possibilidade de serem entrevistados idosos com melhores condições de saúde e, portanto, menor risco de fragilização.

Por outro lado, o maior risco de fragilização nesta pesquisa foi associado ao uso dos antibióticos, a presença de múltiplos problemas de saúde e a polimedicação, isto ocorre pelo fato de que os idosos com estas características estão mais propensos ao desenvolvimento de interações medicamentosas e reações adversas, bem como, complicações advindas dos próprios problemas de saúde o que aumenta a chance de internações hospitalares.

Mesmo que a princípio Luchetti et. al. ${ }^{13}$ tenham identificado que o sexo e a idade predisponham à polimedicação, no presente trabalho não se verificou tal associação dessas variáveis com a polimedicação, nem com o risco de fragilização. Isto deve ser mais bem investigado em novas pesquisas com idosos com características diferentes, como os institucionalizados ou aqueles que procuram os serviços de saúde.

\section{CONCLUSÃO}

Observaram-se alta prevalência de polimedicação, uso de medicamentos impróprios, uso de antibióticos, prática da automedicação e uso de plantas medicinais. Esta situação faz com que esses idosos se predisponham a problemas relacionados ao uso de medicamentos e mesmo de planta medicinais.

Mesmo que o risco de fragilização tenha sido calculado sobre uma planilha desenvolvida em outro país, ${ }^{9}$ os dados apontam para uma população com baixo risco, o que é adequado para idosos em ambiente domiciliar. Se na pesquisa os idosos de baixo risco foram $88 \%$, isso condiz com os achados de Veras, que refere $11 \%$ de prevalência para risco de fragilidade - portanto, de acordo com o esperado.

Propõe-se a realização de novas pesquisas que busquem identificar os riscos de fragilização em outras populações de idosos, em especial em ambientes com maior morbidade, como os de pacientes hospitalizados e institucionalizados em locais de longa permanência.

\section{REFERÊNCIAS}

1. Veras R. Em busca de uma assistência adequada à saúde do idoso: revisão da literatura e aplicação de um instrumento de detecção precoce e de previsibilidade de agravos. Cad Saúde Pública 2003; 19: 705-715.

2. Lojoduci DC,et al. Quedas de idosos institucionalizados: ocorrência e fatores associados. Rev Bras Geriatr Gerontol 2010; 13(3): 403-412.

3. Galato D, Silva ES, Tibúrcio LS. Estudo de utilização de medicamentos em idosos residentes em uma cidade do sul de Santa Catarina: um olhar sobre a polimedicação. Ciênc Saúde Colet 2010; 15(6): 2899-2905.

4. Vieira FS. Possibilidades de contribuição do farmacêutico para a promoção da saúde. Ciênc Saúde Colet 2007; 12: 213-220.

5. Fick DM, et al.Updating the beers criteria for potentially inappropriate medication use in older adults. Arch Intern Med 2003; 163: 2716-2724.

6. Tibincoski $\mathrm{KM}$,et al. Uso de medicamentos no tratamento de idosos atendidos em unidade básica de saúde no Sul de Santa Catarina: um olhar sobre o uso de antibacterianos. Arq Cat Med 2007; 36: 12-17.

7. Cascaes EA, Falchetti ML, Galato D. Perfil da automedicação em idosos participantes de grupos da terceira idade de uma cidade do sul do Brasil. Arq Cat Med 2008; 37: 63-69.

8. Negri LSA,et al. Aplicações de um instrumento para detecção precoce e previsibilidade de agravos na população idosa. Ciênc Saúde Colet 2004; 9 (4): 1033-1046. 
9. Pacala JT, Boult C, Boult L. Predictive validity of a questionnaire that identifies older persons at risk for hospital Admission. J Am Geriatrics Society 1995; 43: 374-377.

10. Dutra MM,et al. Validade preditiva de instrumento para identificação do idoso em risco de hospitalização. Rev Saúd Publica 2011; 45(1): 106-112.

11. World health organization. Complete anatomic therapeutic chemical index. [ Accessed in 10 Mar 2011]. Available from URL: http:// www.whocc.no/atc_ddd_index/.

12. Zamparetti FO, Luciano LTR, Galato D. Estudo de utilização de medicamentos em uma instituição de longa permanência para idosos do Sul de Santa Catarina. Lat AmJ Pharm 2008; 27(4): 553-559.
13. Luchetti G, Granero AL, Pires SL, Gorzoni ML. Fatores associados à polifarmácia em idosos institucionalizados. Rev Bras Geriatr Gerontol 2010; 13(1): 51-58.

14. Organización Mundial de la Salud. Promoción del uso racional de medicamentos: componentes centrales. Genebra: OMS; 2002.

15. Rozenfeld S, Fonseca MJM, Acurcio FA. Drug utilization and polypharmacy among the elderly: a surveyin. Rev Panam Salud Publica 2008; 23: 34-43.

16. Coutinho ESF, Silva SD. Uso de medicamentos como fator de risco para fratura grave decorrente de queda em idosos. Cad Saúde Pública 2002; 18(5): 1359-1366. 